\title{
WHERE ARE THE BUTTERFLY GARDENS?
}

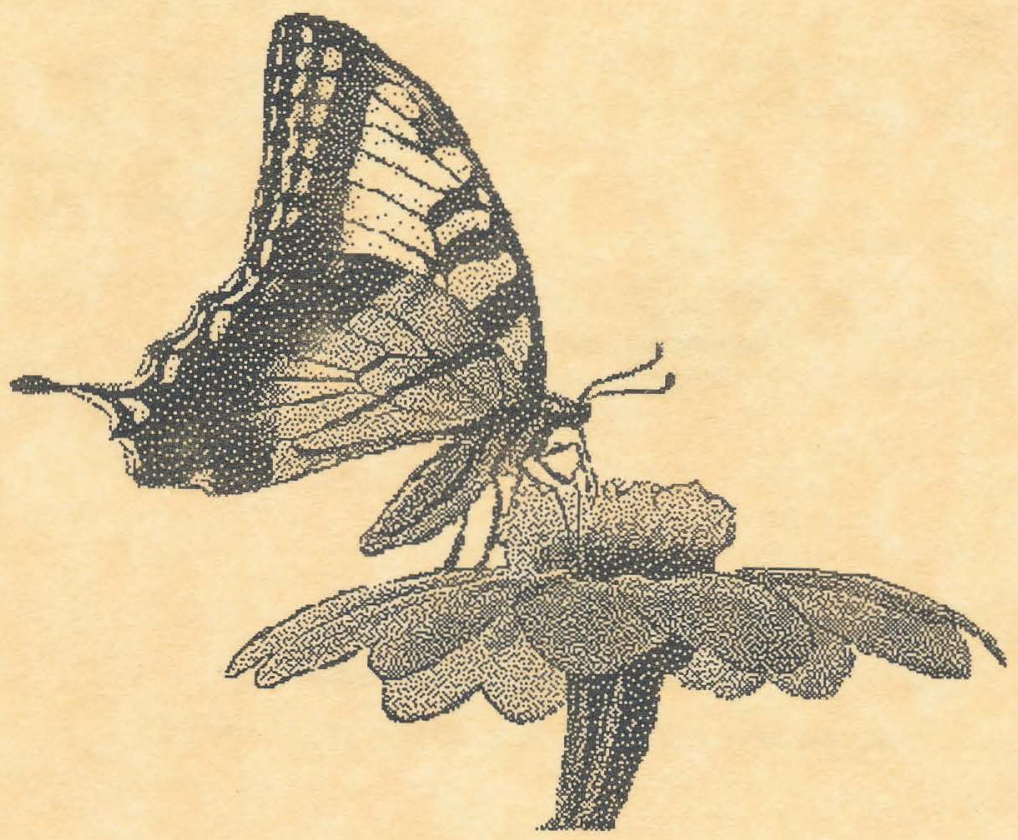




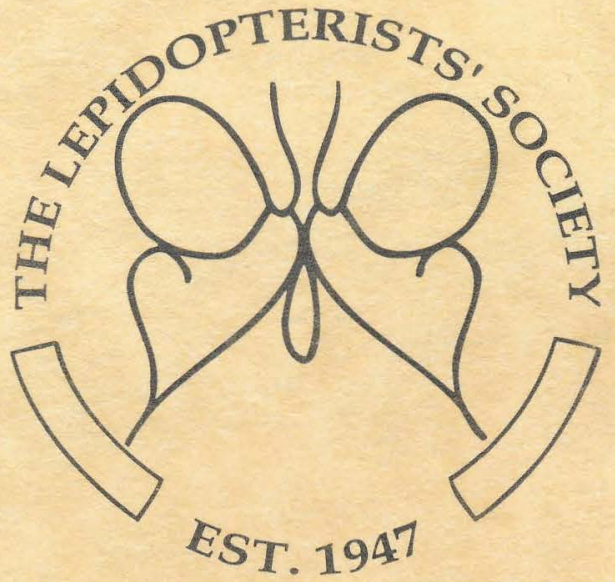

An international organization of amateurs and professionals dedicated to the study of

Butterflies and Moths.

\author{
(c) The Lepidopterists' Society \\ 1200 John Street \\ Manhattan Beach, CA 90266-2608
}


The list of butterfly gardens has been put together for travelers who enjoy butterfly watching, gardening, photography, and as a resource for people who are planning a butterfly garden. I was frequently asked if there were other gardens in a specific area where information on nectar and host plants and local butterflies could be shared.

In some states, schools had planted butterfly gardens with great success. In one area of Florida there are forty schools enjoying their butterfly gardening. They were not included in this list as most of them are inaccessable to the public.

Many of the Nature and Environmental Centers have very small butterfly gardens. Telephone numbers have been included, so do call these places before visiting as there may have been some changes, including deer having eaten everything! Also, so much depends on the focus of the teacher/ naturalist at each place. But it is worth remembering they also offer many other things besides butterfly gardening, such as nature trails and bird watching, etc.

After a year collecting butterfly garden information, I feel that I have new friends throughout North America and I wish I could spend the next year travelling around the country! Many, many thanks to everyone who responded to my letters.

(C) Jane Ruffin

October 1993

The list should not be used for commercial purposes. 


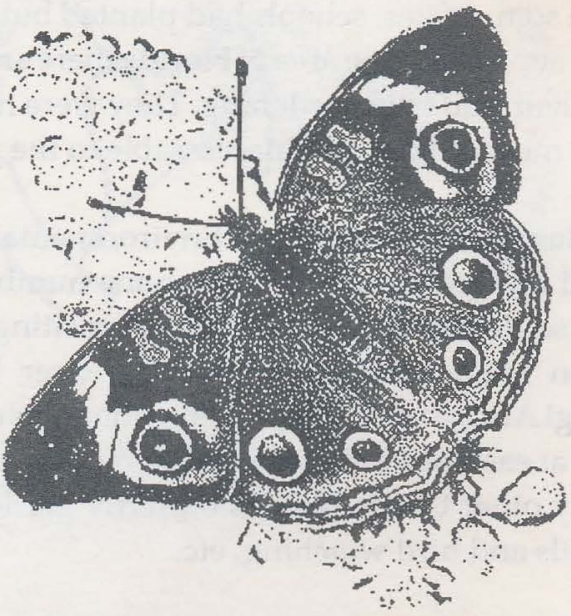




\section{ALABAMA}

Wheeler National Wildlife Refuge

Route 4 Box 250

Decatur, AL 35603

Small butterfly garden planted with flowers and shrubs to attract butterflies.

\section{ARKANSAS}

Pinnacle Mountain State Park Visitor Center 1901 Pinnacle Valley Road

Roland, AR 72135

tel. 501 868-5806

Butterfly gardens planted with host and nectar flowers and shrubs. Butterfly related programs. 


\section{ARIZONA}

Arizona-Sonora Desert Museum

2041 N. Kinney Road

Tucson, AZ 855743

tel. 602885-1380

Backyard habitat attracting butterflies and moths. Workshop for members.

Sonoran Arthropod Studies, Inc. (SASI)

PO Box 5624

Tucson, AZ 85703

tel. 602883-3945

Insect programs. Butterfly garden planned. 


\section{CALIFORNIA}

Marine World Africa USA

Marine World Parkway

Vallejo, CA 94589

tel. $707644-4000$ ext. 270

Butterfly World. Glasshouse with free-flying butterflies.

Daily tours.

San Diego Wild Animal Park

15500 San Pasqual Valley Road

Escondido, CA 92027-9614

tel. 619480-9573

Glasshouse planted with tropical vegetation. Butterflies from April to September. Programs.

San Francisco Zoological Society

Sloat Blvd. / Great Highway

San Francisco, CA 94132

tel. 415 753-7053

Insect Zoo with demonstration butterfly garden.

University of California at Santa Cruz

Santa Cruz, CA 95064

tel. 408 427-2998

Arboretum, with butterfly garden planned. Daily walks. 


\section{COLORADO}

Rocky Mountain Butterfly Consortium

PO Box 377

Westminster, CO 80030-2041

tel. 303 936-2041

Butterfly House opening 1994/95.

Butterfly Garden

3455 Nuckholls Avenue

Pueblo, CO 81005

tel. 719561-8686

Butterfly garden planted with butterfly attracting plants and interpretive signs. Programs. 


\section{DELAWARE}

Ashland Nature Center

Delaware Nature Society

PO Box 700

Hockessin, DE 19707

tel. 302 239-2334

Meadows managed to attract butterflies. 


\section{FLORIDA}

"Butterflies in Flight"

1100 Hollygate Lane

Naples, FL 33940-3847

tel. 813 263-6895

Office address of the travelling enclosed exhibit of native butterflies and plants.

BUTTERFLY WORLD

Tradewinds Park

3600 West Sample Road

Coconut Creek, FL 33073

tel. 305 977-4400

First butterfly house in the U.S. Enclosed garden with native and tropical butterflies. Outdoor butterfly garden. Programs and plants.

Briggs Nature Center

401 Shell Road

Naples, FL 33692

tel. $813775-8569$

Butterfly garden and Nature Center 
Museum of SCIEnce And Industry (MOSI)

4801 East Fowler Avenue

Tampa, FL 33617-2099

tel. 813 985-1914

"Butterfly Encounter" Butterfly exhibit in the museum planted with host and nectar flowers and shrubs. Programs.

Selby Botanical Gardens

811 S. Palm Avenue

Sarasota, FL 34236

tel. $813366-5731$

Demonstration garden for native butterflies. Programs.

Wings OF WONDER / Cypress Gardens

PO Box 1

Cypress Gardens, FL 33884

tel. $813324-4826$

Glass Conservatory with native and tropical butterflies and plants. Outdoor butterfly garden. Programs.

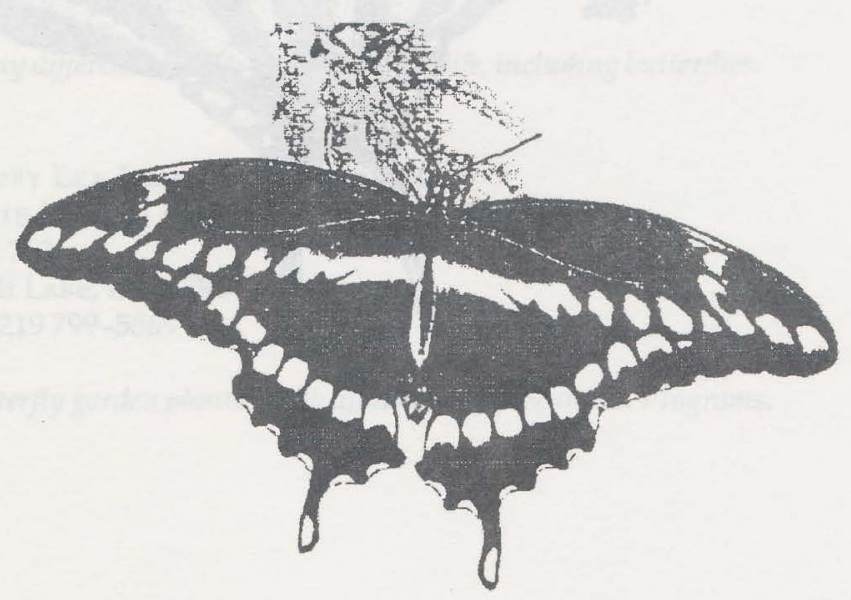




\section{GEORGIA}

The Day Butterfly Center

Callaway Gardens

Pine Mountain, GA 31822-2000

tel. 706 663-2281

Glass Conservatory with native and tropical butterflies. Outside butterfly garden. Programs.

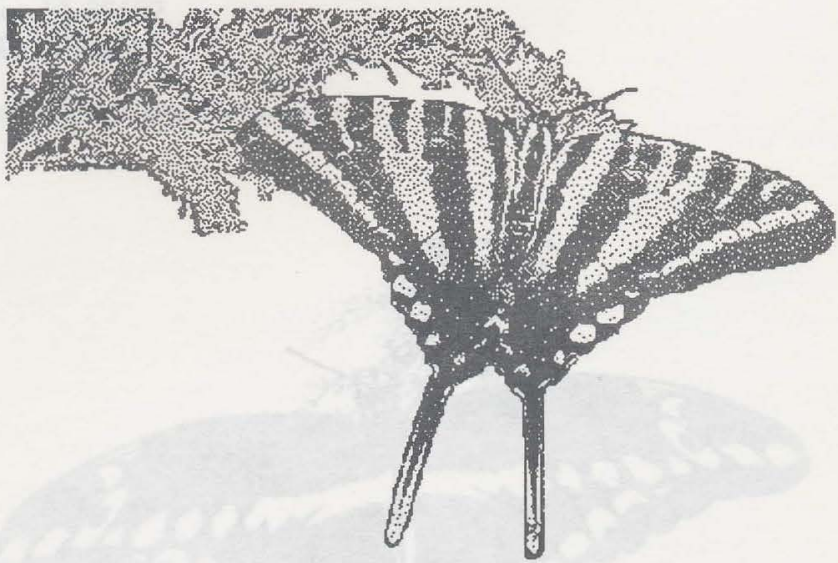




\section{IDAHO}

Morrison Knudson Nature Center Idaho Department of Fish and Wildlife 600 S. Walnut

Boise, ID 83707

tel. 208 334-2225

Garden to attract hummingbirds and butterflies with focus on habitat.

\section{INDIANA}

Dobbs Park Nature Center

5050 East Poplar Drive

Terre Haute, IN 47803-9756

tel. 812 887-1095

Hummingbird and butterfly garden with annual perennial plants.

INDIANAPOLIS ZOO

1200 W. Washington Street

Indianapolis, IN 46222

tel. 317 630-2001

Many different habitats attracting wildlife, including butterflies.

Merry Lea Learning Center and

State Nature Preserve of Goshen College

Box 263

Wolf Lake, IN 46796

tel. 219 799-5869

Butterfly garden planted with annuals and perennials. Programs. 


\section{IOWA}

South Bluff Nature Center

Bellevue State Park

Bellevue, IA 52031

tel. $319872-4019$

Butterfly garden with nectar and host plants. Prairie site and wildlife food plots. Programs. 


\section{KANSAS}

Botanica, The Wichita Gardens

701 Amidon

Wichita, KS 67203

tel. 316 264-0448

Butterfly garden featuring nectar and host plants. Programs.

TOPEKA ZOO

635 Gage Avenue

Topeka, KS 66606

tel. $913272-5821$

Butterfly garden with nectar and host plants. 


\section{LOUISIANA}

Acadiana Park Nature Center c/o Lafayette National History Museum 637 Girard Park Drive

Lafayette, LA 70503

tel. 318 235-6181

Wildflower demonstration garden to attract hummingbirds and butterflies.

Baton Rouge Garden Center 7950 Independence Center

Baton Rouge, LA 70806

tel. 504 928-2270

Small demonstration garden.

Bossier Parish Nature Center

Linton Road

Benton, LA 71006

tel. 318 965-0679

Wildflower meadow with nature trail.

Greater Baton Rouge Zoo

3601 Thomas Road

PO Box 60

Baker, LA 70704

tel. 504 775-3877

Small demonstration garden. 
Louisiana Nature and Science Center PO Box 870610

New Orleans, LA 70187-0610

Demonstration wildflower, butterfly and hummingbird garden.

Nature's Treasures

5555 Government Street

Baton Rouge, LA 70806

tel. 504 925-8894

Small demonstration garden for butterflies and hummingbirds.

New Orleans Botanical Garden

Butterfly Walk

1 Palm Drive

New Orleans, LA 70124

tel. 504 483-9386

Small demonstration garden.

Walter B. Memorial Nature Park

8012 Blanchard Furrh Road

Shreveport, LA 71107

tel. 318929-2806

Hummingbird and butterfly garden. 


\section{MARYLAND}

Black Hill Regional Park Visitor Center

20926 Lake Ridge Drive

Boyds, MD 20841

tel. 301 916-0220

"Wild Wings" garden for hummingbirds and butterflies.

BROOKSIDE GARDENS

1500 Glenallen Avenue

Wheaton, MD 20902

tel. 301 949-8230

Demonstration butterfly garden.

Brookside Nature Center

1400 Glenallen Avenue

Wheaton, MD 20902

tel. 301 946-2071

Demonstration garden.

Clearwater Nature Center

Cosca Regional Park

11000 Thrift Road

Clinton, MD 20735

tel. 301 297-4575

Prince George butterfly garden planted with nectar and host plants. 
Horsehead Wetlands Center

600 Discovery Lane

PO Box 519

Grasonville, MD 21638

tel. 410 827-6694

Demonstration garden for hummingbirds and butterflies.

Irvine Natural Science Center

St. Timothy's School

Stevenson, MD 21153

tel. $301484-2413$

Small demonstration garden.

Jug Bay Wetlands Sanctuary

1361 Wrighton Road

Lothian, MD 20711

tel. 410 741-9330

Small demonstration garden.

Meadowside Nature Center

5100 Meadowside Lane

Rockville, MD 20853

tel. 301 924-4141

Small demonstration garden. 


\section{MASSACHUSETTS}

"Butterfles in Flight" on Martha's Vineyard Circuit Avenue Extension

Oak Bluffs, MA 02557

tel. 508 693-4006

Seasonal enclosed exhibit with native butterflies.

The Butterfly Place

Papillion Park

120 Tyngsboro Road

Westford, MA 01886

tel. 508392-0955

Large glass atrium with native butterflies and an outdoor butterfly habitat.

Massachusetts Audubon Society

Drumlin Farm Sanctuary

Route 117 S. Great Road

Lincoln, MA 01773

tel. 617 259-9661

Butterfly garden.

LOREne MELVIN

27 Lafayette St

Arlington, MA 02174

Private butterfly garden. BY APPOINTMENT ONLY. 
Moose Hill Wildlife Sanctuary

Massachusetts Audubon Society

Sharon, MA 02067

tel. 617 784-5691

Butterfly garden and habitat.

Patricia Dennett Memorial Butterfly Garden

South Shore Natural Science Center

Jacobs Lane Norwell, MA 02061

tel. $617878-8039$

Butterfly garden planted with nectar, host and native plants.

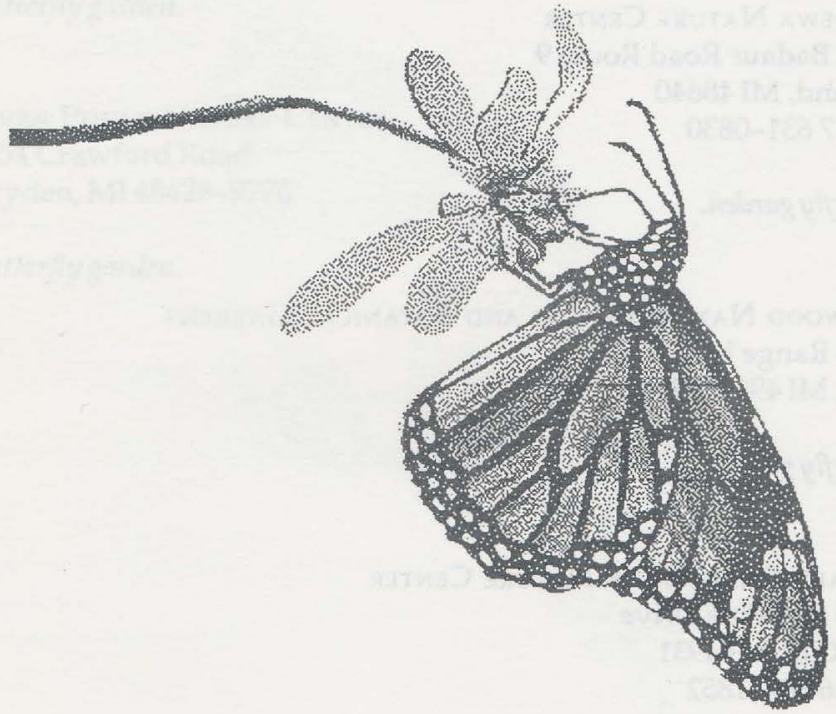




\section{MICHIGAN}

Botany and Plant Pathology Greenhouse

Michigan State University

Circle Drive

East Lansing, MI 48824

tel. 517 355-0229

Exhibit demonstrating the life cycle of butterflies with host and nectar plants.

"Butterfles in Flight"

Mackinac Island Butterfly House

Sawyer's Greenhouse

1308 McGulpin

Mackinac Island, MI 49757

tel. $906847-3972$

Seasonal enclosed exhibit with native butterflies.

Chippewa Nature Center

400 S. Badaur Road Route 9

Midland, MI 48640

tel. $517631-0830$

Butterfly garden.

Fernwood Nature Center and Botanical Gardens 13988 Range Line Road

Niles, MI 49120-9042

Butterfly garden.

Howard Christensen Nature Center 16160 Red Pine Drive

Kent City, MI 4931

tel. 616 887-1852

Butterflygarden. 
Kalamazoo Nature Center

7000 North Westnedge Avenue

Kalamazoo, MI 49004

tel. 616381-1574

Hummingbird and butterfly demonstration garden.

Horticultural Demonstration Gardens

Michigan State University

Wilson Road and Bogue St.

Michigan State University Campus

East Lansing, MI 48824

Children's butterfly garden.

Sarrett Nature Center

Michigan Audubon Society

2300 Benton Harbor, MI 49022

tel. 616 927-4832

Butterfly garden.

Seven Ponds Nature Center

3854 Crawford Road

Dryden, MI 48428-9776

Butterfly garden. 


\section{MINNESOTA}

Minnesota Zoo

13000 Zoo Boulevard

Apple Valley, MN 55124

tel. 612 431-9381

Backyard exhibit to attract wildlife including hummingbirds and butterflies.

\section{MISSISSIPPI}

The Jackson Zoological Park, Inc. 2918 W. Capitol Street

Jackson, MS 39201

Demonstration butterfly garden.

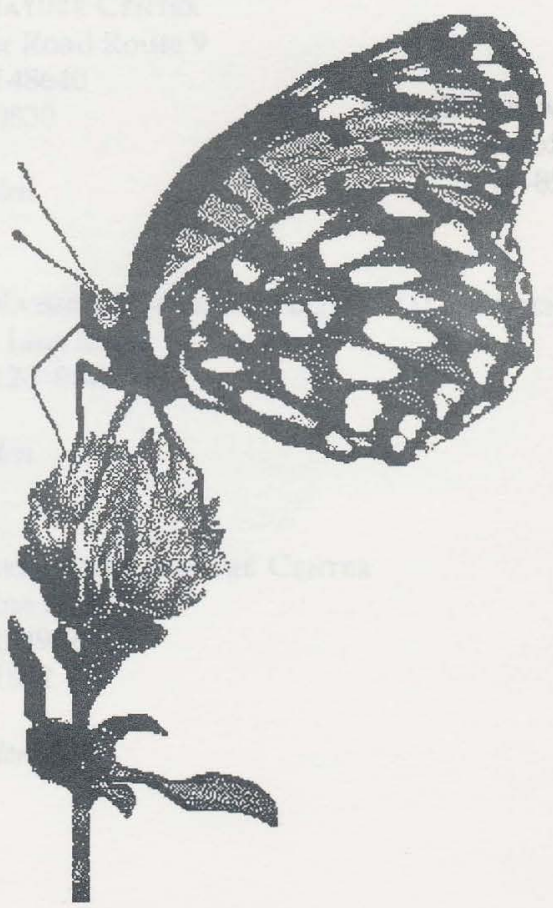




\section{MISSOURI}

Kansas City Zoo

6700 Zoo Drive

Kansas City, MO 64132

tel. 816 333-7405

Butterfly garden.

Powder Valley Nature Center

Missouri Department of Conservation

11715 Cragwold Road

Kirkwood, MO 63122

tel. 314 821-8442

Butterfly garden.

Runge Conservation Nature Center

Missouri Department of Conservation

Highway 179

Jefferson City, MO 65102

tel. 314 526-5544

Butterfly and hummingbird garden.

Springfield Nature Center

Missouri Department of Conservation

4600 South Chrisman

Springfield, MO 65804

tel. $417882-4237$

Native plantings to encourage hummingbirds and butterflies.

Zoo of ST. Louis

Forest Park

St. Louis, MO 63110

tel. 314 781-0900

Butterfly garden. 


\section{NEBRASKA}

Folsom Children's Zoo and Botanical Garden 1222 S. 27th

Lincoln, NE 68502

tel. 402 475-6741

Hummingbird and butterfly garden. Programs.

Pioneers Park Nature Center

Lincoln Parks and Recreation

2740 A St.

Lincoln, NE 68502

tel. 402 441-7895

Butterfly gardens including nectar and host plants.

\section{NEW JERSEY}

Cape May Bird Observatory

PO Box 3

Cape May Point, NJ 08212

tel. $609884-2736$

Demonstration butterfly and hummingbird garden.

Flat Rock Brook Nature Center

443 Van Nostrand Avenue

Englewood, NJ 07631

tel. 201 567-1265

Butterfly garden.

Joan Tuite Butterfly Garden

Davies Arboretum

49 Anderson Avenue

Demarest, NJ 07627

Flowers and shrubs to attract butterflies. 
Leamings Run Botanical Garden's and Colonial Farm

1845 Route 9 North

Swainton, NJ 08210

tel. 609 465-5871

Hummingbird and butterflies encouraged in the gardens.

Pequest Trout Hatchery and Natural Resource Center RR1 Box 389

Oxford, NJ 07863

tel. $201637-4125$

Butterfly garden.

Plainsboro Library

Plainsboro Road

Plainsboro, NJ 08536

tel. 6092752897

Hummingbird and butterfly garden.

St. Mary's Roman Catholic Church Highway 34 and Phalanx Road

Cotts Neck, NJ 07722

Butterfly garden.

Trailside Nature and Science Center Coles Avenue and New Providence Road Mountainside, NJ 07092

tel. 908 789-3670

Butterfly garden. 


\section{NEW MEXICO}

Living Desert State Park

PO Box 100

Carlsbad, NM 88220

tel. $505887-5516$

Botanical garden with different zones planted with native flowers.

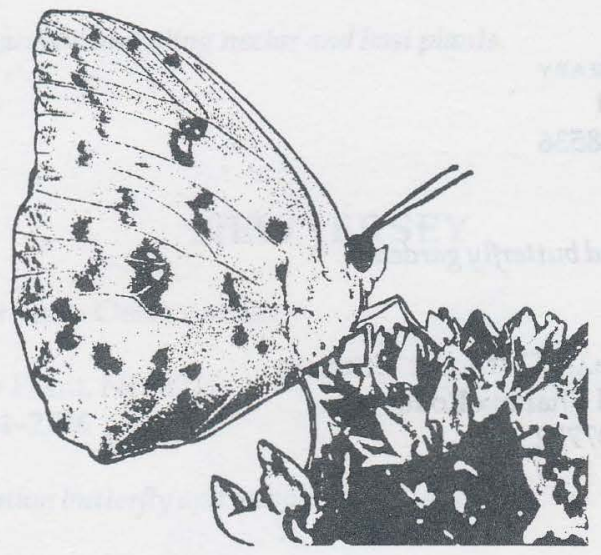




\section{NEW YORK}

Adirondack Park Visitor Interpretive Center

PO Box 101

Newcomb, NY 12852

tel. 518 582-2000

Butterfly garden with native plantings to attract Adirondack butterflies.

Beaver Meadow Audubon Center

1610 Welch Road

North Java, NY 14113

tel. 716 457-3228

Butterfly garden and meadows.

Jamaica Bay Wildlife Refuge

Gateway National Recreation Area

Floyd Bennet Field

Brooklyn, NY 11234

tel. $718474-0613$

Butterfly garden.

New York Audubon Society

Hollyhock Hollow Sanctuary

131 Rarick Road

Selkirk, NY 12158

tel. 518 767-9051

Demonstration butterfly garden including nectar and host plants.

Rogers Environmental Education Center

PO Box 716 Route 80W

Sherburne, NY 13460-0716

tel. 607 674-4017

Perennial butterfly garden. Programs.

Ross Park Zoo

185 Park Avenue

Binghamton, NY 13903

tel. 607 724-5461

Butterfly garden. 


\section{NORTH CAROLINA}

Greensboro Arboretum

West Market Street and Ashland Drive

Greensboro, NC 27403

tel. 919373-2558

Butterfly garden including host and nectar plants.

North Carolina Zoological Park

Route 4 Box 83

Asheboro, NC 27203

tel. 919879-7000

Butterfly and hummingbird garden.

UNC Charlotte Botanical Gardens

Biology Department

University of North Carolina

Charlotte, NC 28223

tel. 704 547-4055

Plants to attract butterflies.

Western North Carolina Nature Center

75 Gashes Creek Road

Asheville, NC 28805

tel. 704 298-5600

Butterfly garden featuring plants native to the southern Appalachian Mountain region. Nectar and host plants.

WestPoint ON THE ENO

5101 N. Roxboro Road

Durham, NC 27704

tel. 919471-1623

Demonstration butterfly garden. 


\section{OHIO}

Cincinnati Zoo znd Botanical Garden

34400 Vine Street

Cincinnati, OH 45220-1399

tel. 513 281-4701

Insectarium with glass conservatory. Outdoor butterfly garden. Programs.

Farbach-Werner Preserve / Hamilton County Nature Preserve Colerain and Poole Road

Cincinnati, OH 45251

tel. 513 385-4811

Demostration garden including host and nectar plants.

Hearts and Flowers Butterfly Farm

3977 Condit Road

Sunbury, OH 43074

tel. 614 965-2133

Display garden and nursery.

Highbanks Metro Parks

9466 N. High Street

Powell, OH 43081

tel. 614 846-7167

Small perennial butterfly garden. Programs.

Northgate Butterfly Park

Columbus, OH 43229

tel. 614 882-6931

Butterfly garden including nectar and host plants.

Penitentiary Glen Nature Center

A Lake Metroparks Facility

8668 Kirtland-Charden Road

Kirtland, OH 44094

tel. 216 256-1404

Butterfly garden planted with annual and perennial flowers.

Toledo Botanical Gardens

5403 Elmer Drive

Toledo, OH 43615

tel. 419536-8365

Small butterfly garden. 


\section{OKLAHOMA}

Myriad Gardens

100 Myriad Gardens

Oklahoma City, OK 73102

tel. 405 297-3995

Butterfly garden.

Oklahoma City Zoological Park

2101 N.E. 50 th

Oklahoma City, OK 73111-7199

tel. 405 424-3344

Butterfly garden with nectar and host plants.

The Tulsa Zoo

5701 East 36th Street North

Tulsa, OK 74115

tel. 918 596-2415

Butterfly garden in the Children's Zoo with nectar and host flowers and shrubs. 


\section{OREGON}

Macadam Bay Club

Macadam Avenue

c/o Portland Park Bureau

1120 S.W. 5th Street

Portland, OR 97201

tel. 823 796-5122

Butterfly park with native plants.

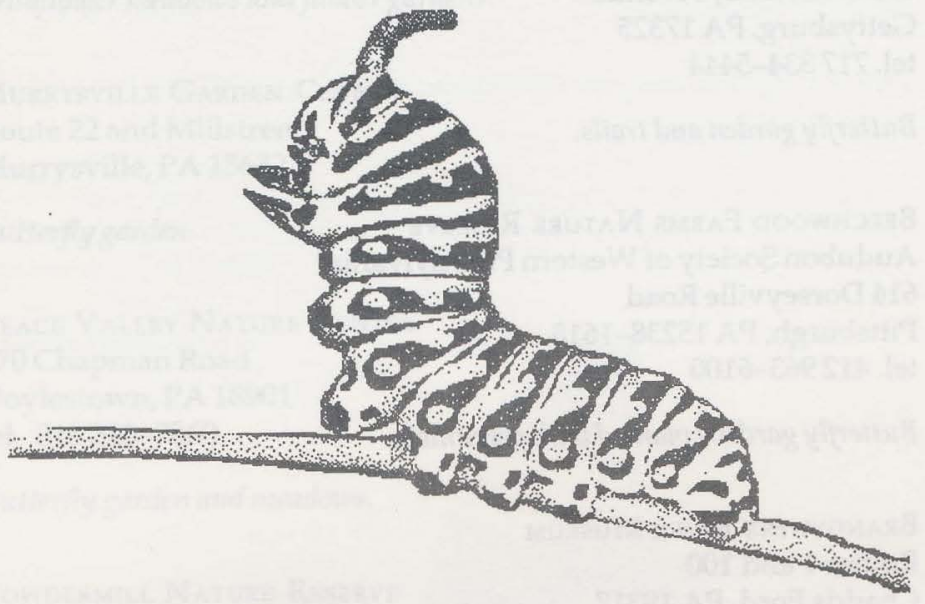




\section{PENNSYLVANIA}

Alloway Gardens and Herb Farm

456 Mud College Road

Littlestown, PA 17340

tel. 717 359-4363

Demonstration butterfly garden and nursery.

Bald Eagle State Park

R.D. \#1 Box 56

Howard, PA 16841

tel. 814 625-2775

Butterfly trail.

Battlefield Butterfly Garden

135 Doubleday Avenue

Gettysburg, PA 17325

tel. 717 334-5444

Butterfly garden and trails.

Beechwood Farms Nature Reserve

Audubon Society of Western Pennsylvania

614 Dorseyville Road

Pittsburgh, PA 15238-1618

tel. 412963-6100

Butterfly gardens planted with perennials.

Brandywine River Museum

Routes 1 and 100

Chadds Ford, PA 19317

tel. 215388-7601

Native wildflowers and meadows.

Churchville Nature Center

501 Churchville Lane

Churchville, PA 18966

tel. 215 357-4005

Butterfly garden and trails. 
The John J. Tyler Arboretum

515 Painter Road

Lima, PA 19060

tel. 215 566-9133

Butterfly garden.

LONGWOOD GARDENS

Kennet Square, PA 19348

tel. 215388-6741

Hummingbird and butterfly garden.

Morris Arboretum of the University of Pennsylvania 9414 Meadowbrook Avenue

Philadelphia, PA 19118

215247-5777

Wildflower meadows and flower gardens.

Murrysville Garden Club

Route 22 and Millstreet

Murrysville, PA 15632

Butterflygarden.

Peace Valley Nature Center

170 Chapman Road

Doylestown, PA 18901

tel. $215345-7860$

Butterfly garden and meadows.

Powdermill Nature Reserve

Star Route South

Rector, PA 15677

tel. 412 593-6105

Butterflygarden. 


\section{RHODE ISLAND}

Newport Butterly Farm

The Rare Butterfly Company

1151 Aquidneck Avenue, \#409

Middletown, RI 02340

tel. 401 846-3148

Butterfly garden. BY APPOINTMENT ONLY

\section{SOUTH CAROLINA}

The Butterfly BARN

PO Box 214/816 Pinckney Street

McClellanville, SC 29458

tel. 803-887-3938

Butterly flight atrium planted with native flowers and trees.

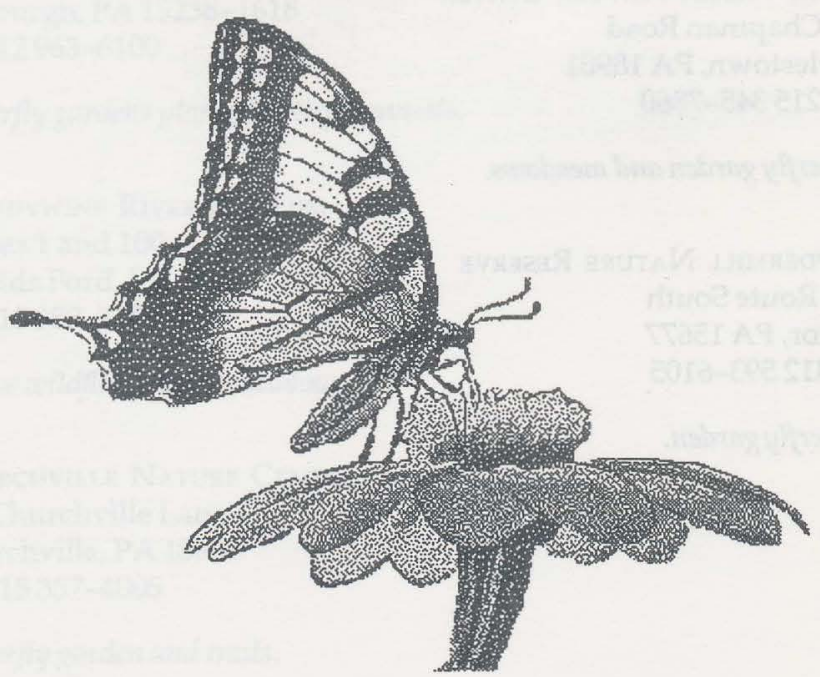




\section{SOUTH DAKOTA}

Great Plains and Delbridge Museum

805 S. Kiwanis Avenue

Sioux Falls, SD 57104-3741

tel. 605 339-7003

Perennial and annual butterfly garden.

\section{TENNESSEE}

Ijams Nature Center

2915 Island Howe Avenue

Knoxville, TN 37920

tel. 615 573-7183

Wildflower meadows and plants for butterflies.

St. Francis of Assisi Catholic Church

St. Francis Lane and River Road

Townsend, TN 37882

Small butterfly garden. 


\section{TEXAS}

Butterfly / Tropical Rainforest Center

Houston Museum of Natural Science

One Hermann Circle Drive

Houston, TX 77030

tel. 713 639-4678

Opening 1994.

Dallas Zoo

621 East Clarendon

Dallas, TX 75205

tel. 214 670-6772

Butterfly garden with nectar and host plants.

Mercer Arboretum and Botanic Gardens

22306 Aldine Westfield Road

Humble, TX 77338

tel. $713443-8731$

Hummingbird and butterfly garden including nectar and host plants. Programs.

Mary Alice Perkins Bland Garden

Dallas Nature Center

7171 Mountain Creek Parkway

Dallas, TX 75249

tel. 214 296-1955

Small butterfly garden.

San Antonio Zoo

3903 North St. Mary's

San Antonio, TX 78212

tel. 512 734-7184

Hummingbird and butterfly garden including nectar and host plants.

Zilker Botanical Garden

Austin Area Garden Center

2220 Barton Springs Road

Austin, TX 78746

tel. $512477-8672$

Butterfly trail. 


\section{VIRGINIA}

Riverbend Nature Center

8814 Jeffery Road

Great Falls, VA 22066

tel. 703 759-3211

Meadows and butterfly garden. Programs.

The Vernon J. Walker Nature Education Center

Naturescaping Garden

11450 Glade Drive

Reston, VA 22090

tel. 703 476-9689

Butterfly and hummingbird garden.

ViRginia Living Museum

$524 \mathrm{~J}$. Clyde Morris Boulevard

Newport News, VA 23601

tel. 804 595-1900

Small butterfly garden. Programs.

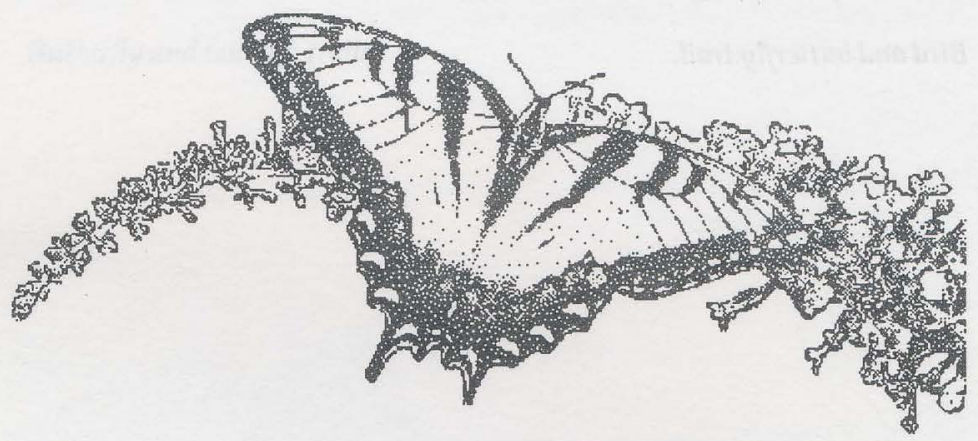




\section{WASHINGTON}

Collman's Chrysilis

13720 23rd Avenue NE

Seattle, WA 98125

tel. 206 364-6966

Butterfly garden. BY APPOINTMENT ONLY.

Lake Hills Greenbelt Ranger Station

15416 S.E. 16th Street

Bellevue, WA 98009

Butterfly garden including nectar and host plants. Programs.

\section{WEST VIRGINIA}

Phymosia Butterfly Garden

Youth Museum of South West Virginia

Beckley, WV 25801

Bird and butterfly refuge.

SPORTSMAN PARK

Elizabeth, WV 26143

Bird and butterfly trail. 


\section{WISCONSIN}

Havenwoods State Forest

6141 N. Hopkins Street

Milwaukee, WI 53209

tel. 414 527-0232

Demonstration butterfly garden including nectar and host plants.

International Crane Foundation

E. 11376 Shady Lane Road

Baraboo, WI 53913

tel. 608356-9462

Prairie restoration including flowers for butterflies.

Milwaukee County / University of Wisconsin Extension 9668 W. Watertown Plank Road

Wauwatosa, WI 53226

tel. 414 475-2200

Demonstration garden with nectar and host plants.

Wehr Nature Center

Whitnall Park Zoo

9701 W. College Avenue

Franklin, WI 53132

Butterfly and wildlife trails. 


\section{BRITISH COLOMBIA}

OKanagon Butterfly World

1190 Stevens Road

Kelowna, British Columbia

Canada V1 Z 1G1

tel. 604 769-4408

Butterfly Conservatory.

Parksville Butterfly World

PO Box 36

Combs, British Columbia

Canada VOR $1 M 0$

tel. 604 248-7026

Butterfly Conservatory.

Victoria Butterfly World, Inc.

PO Box 130

Brentwood Bay, British Columbia

Canada VOS $1 \mathrm{AO}$

tel. 604652-7811

Butterfly Conservatory.

\section{NEWFOUNDLAND}

Memorial University gardens at Oxen Pond

St. Johns, Newfoundland

Canada A1C 557

tel. $709737-8590$

Botanical garden and nature trails planted with flowers and shrubs to attract butterflies and other wildlife.

\section{ONTARIO}

Metro Toronto Zoo

PO Box 280

West Hill, Ontario

Canada M1E 4R5

Enclosed area for free-flight butterflies. 
The North American Butterfly Association NABA

39 Highland Avenue

Chappaqua, NY 10514

Young Entomologists' Society, Inc. Y.E.S.

1915 Peggy Place

Lansing, MI 48910-2553

\author{
Xerces Society \\ 10 S.W. Ash Street \\ Portland, OR 97204
}

Written orders only for this book

Where are the Butterly Gardens?

1013 Great Springs Road

Rosemont, PA 19010

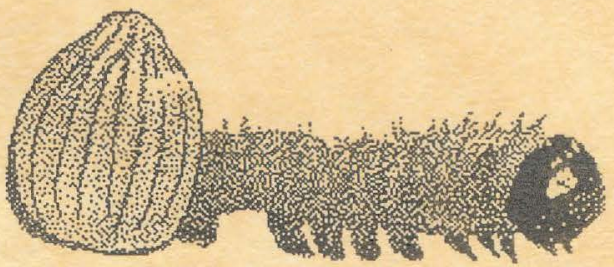





\section{Corrections April 1994}

The Lepidopterists' Society

1900 John Street

Manhattan Beach CA 90266-2608

Rocky Mountain Butterfly Consortium

P.O.Box 377

Westminster CO 80030-0377

Newport Butterfly Farm

1151 Aquidneck Avenue \#409

Middletown RI 02842

tel. $401 \quad 849-9519$ 